\title{
Síntese de Antenas com Refletor Principal Esférico para Geração de Feixe Colimado ${ }^{1}$
}

\author{
J. R. Bergmann e J. C. W. A. da Costa \\ Centro de Estudos em Telecomunicações \\ Pontifícia Universidade Católica do Rio de Janeiro
}

\begin{abstract}
Com vistas a sua utilização como antena de pequenas estações de sistemas de satélite, este trabalho investiga o emprego de refletores esféricos em sistemas de duplos refletores offset. Embora o emprego do refletor esférico seja atraente pelos seus custos de fabricação, os projetos anteriores apresentam baixa eficiência. Neste trabalho, é apresentada uma técnica de projeto que incorpora vários parâmetros que definem a geometria, os quais são otimizados para aumentar a eficiência da antena e baixar o envelope de lobos secundários. É apresentada, também, uma análise da polarização dos campos através do sistema permitindo obter o controle do nível de polarização cruzada radiada pela antena. Utilizando as aproximaçōes da Ótica Física para obter os campos radiados, mostra-se que é possível obter níveis de eficiência superiores a $75 \%$, combinados com níveis de polarização cruzada abaixo de $-60.0 \mathrm{~dB}$ e diagramas de lobos laterais abaixo dos envelopes estabelecidos pelo CCIR $(29-25 \log (\theta))$.
\end{abstract}

The design of dual-reflector antennas with a spherical main reflector for small stations is considered. An analysis of the field polarization throughout the system shows that is possible to adjust the reflector to obtain very low cross-polarization. The use of an elliptical main reflector projection is explored in order to enhance efficiency and lower side-lobe levels.

Palavras chave: Antenas de Duplos Refletores, Refletor Esférico, Antenas para Estações Terrestres.

\section{Introdução}

Para evitar interferência entre sistemas de comunicação que utilizam a órbita geoestacionária, é necessário que o diagrama das antenas das estações terrenas produzam um diagrama de lobos secundários abaixo do envelope definido por $29-25 \log (\theta) \mathrm{dBi}$. Para as antenas das estações terrenas que empregam grandes refletores, as estruturas axialmente simétricas são viáveis visto que a energia bloqueada não é crítica para a degradação do desempenho dos lobos laterais. Entretanto, para as antenas das pequenas estações é essencial a utilização de estruturas offset para evitar o bloqueio produzido pelo subrefletor e pelos estais os quais são responsáveis pela elevação dos níveis de lobos laterais e da polarização cruzada.

1 Este trabalho foi desenvolvido no CETUC, PUC-Rio, Rua Marques de São Vicente 225, CEP 22453, Rio de Janeiro. Ele foi financiado pela TELEBRÁS sob contrato PUC/TELEBRÁS JDPqD-415-91. Manuscrito encaminhado em julho de 1993; revisado e aceito em dezembro de 1993. 
A literatura contém vários projetos de sistemas de duplos refletores [1 - 4], salientando-se o trabalho desenvolvido por Cha [1] que obtém uma eficiência de $85 \%$ (medida) através do modelamento de ambos os refletores. Entretanto, a construção destes refletores envolve processos de fabricação dispendiosos, especialmente do refletor principal. Por outro lado, Ramanujan et al [2] consideraram como objeto de estudo uma configuração de duplos refletores que emprega um refletor esférico como refletor principal. Para obter fase uniforme sobre a abertura, é utilizado um subrefletor cuja superfície é uma porção de uma superfície que possui simetria axial [5]. Estes autores consideraram que o refletor principal projeta uma abertura circular com diâmetro $\mathrm{D}=172 \lambda$ (comprimentos de onda), é um alimentador que radia uma onda esférica com diagrama de radiação do tipo $\cos ^{\mathrm{n}} \theta$. Este modelo representa adequadamente o comportamento das cornetas corrugadas usualmente empregadas nestes projetos. Para calcular o campo espalhado pela antena refletora foram utilizadas as aproximações da Ótica Física. Tendo em conta a necessidade de produzir antenas compactas, pode-se concluir, destes resultados, que o aumento de eficiência depende, essencialmente, da utilização de subrefletores maiores. A configuração gregoriana considerada apresentou uma eficiência máxima de $64.1 \%$ associada a um subrefletor com dimensão máxima de $0.254 \mathrm{D}(\approx 47 \lambda$ ). Esta configuração apresenta limitações ao aumento da eficiência devido as distorções na distribuição de campo produzidos pela aberração esférica.

Atraído pelos reduzidos custos de fabricação do refletor esférico para produção em massa, este trabalho explora seu emprego em nova configuração de antena para pequenas estações terrenas. A configuração considerada aqui não impõe condições de simetria para o subrefletor corretor de fase, como foi sugerido no trabalho de Chang e Rusch [6]. Este grau de liberdade adicional introduzido no sistema permite explorar os parâmetros da geometria com o objetivo de reduzir a distorção na distribuição de campos na abertura e, consequentemente, elevar a eficiência e obter maior folga para os primeiros lobos laterais em relação as especificações do CCIR mencionadas anteriormente. No plano de simetria, os efeitos de transbordamento do subrefletor no diagrama de radiação podem ser significantemente reduzidos pelo alinhamento do eixo do alimentador na direção do lóbulo principal.

$\mathrm{Na}$ configuração apresentada em [2], o diagrama de polarização cruzada depende essencialmente do ângulo $2 \theta_{c}$ subentendido pelos raios que incidem no topo e na base do subrefletor ao longo do plano de simetria. O valor máximo desta componente cresce monotonicamente com $\theta_{\mathrm{c}}$ sugerindo a escolha de um alimentador com diagrama estreito como forma de reduzir a polarização cruzada. Por outro lado, a utilização de alimentadores com diagrama estreitos é limitada pelas perdas por transbordamento e pela necessidade de obter-se configurações compactas. Na configuração considerada neste trabalho, estas limitações são superadas. Uma análise da depolarização do campo introduzida pela dupla reflexão ao longo do sistema mostra que é possível ajustar os parâmetros da geometria a fim de compensar a rotação do vetor polarização. Exemplos de geometrias com níveis de polarização cruzada inferior a -60 dB são apresentadas. 
Para obter a forma do subreflector corretor de fase, esta investigaçāo apresenta uma técnica de síntese baseada nos princípios da Ótica Geométrica associada a notação complexa apresentada por Westcott [7].

\section{Modelamento do Subreflector}

Na referência [7], Westcott apresentou uma técnica de síntese de sistemas de duplos refletores, onde os pontos $(x, y)$ sobre a abertura no plano $\mathrm{z}=0$ são representados pela variável complexa $\tau=x+$ iy (ver Fig. 1).

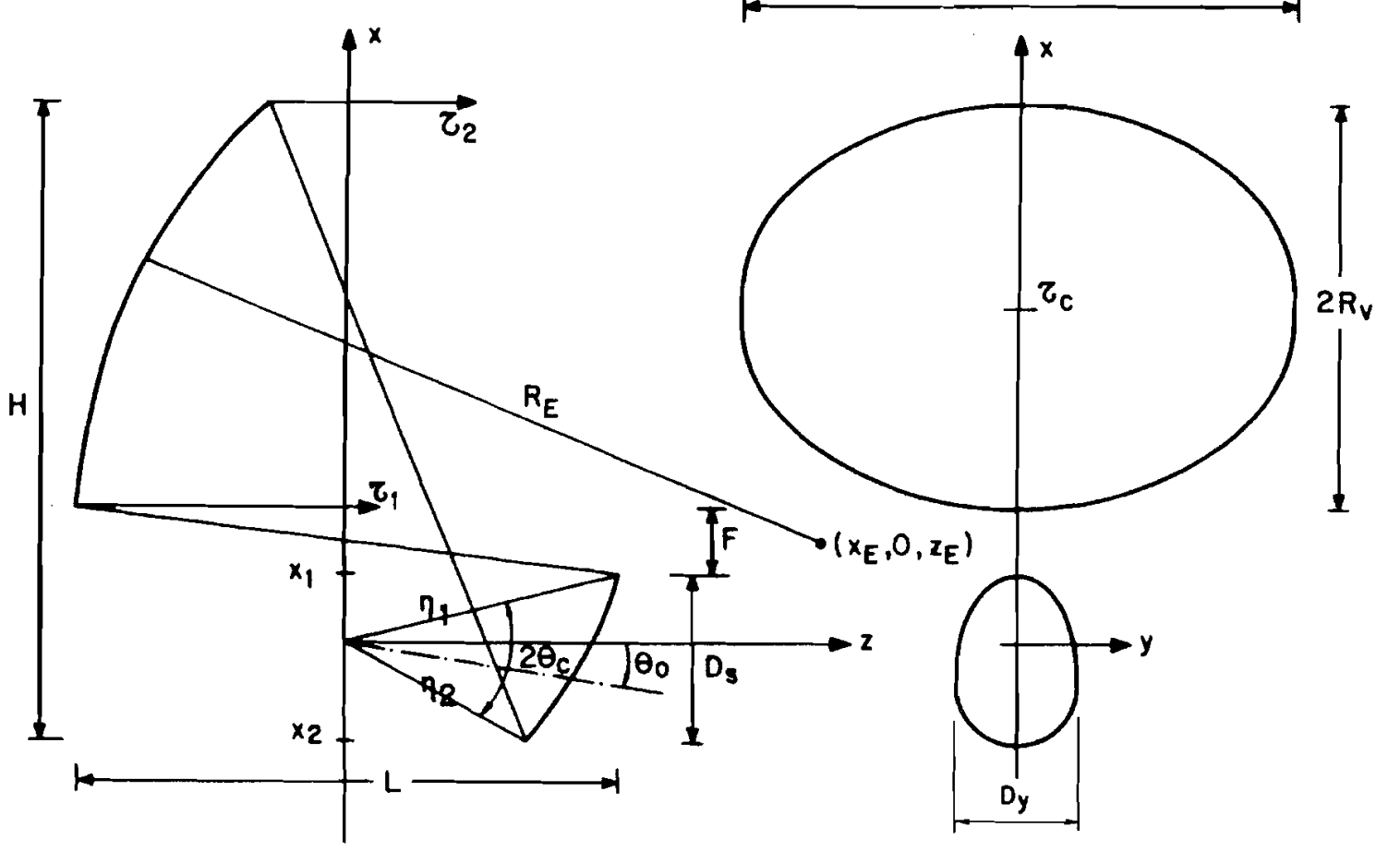

Figura 1 - Geometria gregoriana de duplos refletores

A direção $(\theta, \phi)$ dos raios emitidos por uma fonte pontual sobre a origem é representada pela variável complexa $\eta=\cot (\theta) \exp (i \phi)$. Esta técnica tem sido empregada para $o$ modelamento simultâneo de ambos os refletores a fim de obter uma densidade de potência $G(\tau)$ pré-especificada na abertura quando o sistema é iluminado por uma fonte pontual com diagrama de radiação $I(\eta)$.

O modelamento dos refletores é obtido pela solução de equação diferencial parcial não linear (Monge-Ampere) formulada em termos de função real M ( $\tau$ ) e escrita como 


$$
\left[\left|M_{\tau \tau}-M_{\tau}^{2}\right|^{2}-M_{\tau \bar{\tau}^{2}}\right]=\left[\left|M_{\tau}\right|^{2}\left(1+|\eta|^{2}\right)\right]^{2} I(\eta) /[4 G(\tau)]
$$

onde $\mathrm{M}(\tau)$ é associada a coordenada $\mathrm{z}(\tau, \bar{\tau})$ do refletor principal

$$
\mathrm{M}(\tau)=-\ln \left(2\left[\mathrm{z}(\tau) / \ell_{0}\right]+(|\tau| / \ell)^{2}-1\right)
$$

sendo $\ell_{0}$ o caminho ótico total que o raio percorre da fonte pontual sobre a origem até o plano da abertura. A variável $\bar{\tau}$ denota o complexo conjugado de $\tau$ e $\mathrm{M}_{\tau}, \mathrm{M}_{\tau \tau}, \mathrm{M}_{\bar{\tau} \bar{\tau}}$ representam as derivadas parciais de primeira e segunda ordem de $\mathrm{M}(\tau)$ com respeito a $\tau$ e $\bar{\tau}$.

A utilização desta função $\mathrm{M}(\tau)$ resulta em uma expressão simples para o mapeamento $\eta(\tau)$

$$
\eta(\tau)=\left[\tau+1 / M_{\tau}\right] / l_{\infty}
$$

sendo a superfície do subrefletor descrita pelo vetor $\vec{r}(\tau)$, o qual, em coordenadas esféricas, é dado por

$$
\overrightarrow{\mathrm{r}}(\tau)=\frac{\ell_{0} \exp [\mathrm{M}(\tau)]}{\exp [\mathrm{M}(\tau)]+\left|\mathrm{M}_{\tau}{ }^{2}\right|}\left[(\eta+\bar{\eta}), \mathrm{i}(\bar{\eta}-\eta),\left(|\eta|^{2}-1\right)\right]
$$

Em vez de resolver a equação diferencial (1) para determinar $M(\tau)$, esta formulação pode ser empregada para encontrar a forma do subrefletor corretor associado a um refletor principal específico descrito pela função $\mathrm{M}(\tau)$ como definido por (2). Para um refletor esférico de raio $\mathrm{R}_{\mathrm{E}}$ e centro no ponto $\left(\mathrm{x}_{\mathrm{E}}, 0, \mathrm{z}_{\mathrm{E}}\right)$ sobre o plano de simetria $\mathrm{y}=0$, a função $\mathrm{M}(\tau)$ é obtida substituindo $\mathrm{z}(\tau)=\left[\mathrm{R}_{\mathrm{E}}^{2}-\left|\tau-\mathrm{x}_{\mathrm{E}}\right|^{2}\right]^{1 / 2} \mathrm{em}(2)$, resultando em

$$
\exp [-M(\tau)]=\left\{2\left[\mathrm{z}_{\mathrm{E}}-\mathrm{s}(\tau)\right] / \ell_{0}-\left(\tau / \ell_{0}\right)^{2}-1\right\}
$$

onde $s(\tau)=\left[R_{E}^{2}-\left|\tau-x_{E}\right|^{2}\right]^{1 / 2}$ para os raios cruzando a abertura na direçāo do eixo z-positivo como indicado na Fig.1. Desta forma, o sistema é definido pela associação dos parâmetros $\ell_{0}, \mathrm{x}_{\mathrm{E}}, \mathrm{z}_{\mathrm{E}}$ com as especificações do projeto. 
Aqui, estes parâmetros são determinados forçando três condições para os raios que cruzam a abertura nos pontos $\tau_{1,2}=\tau_{C} \pm R_{V}$ sobre o plano de simetria. Com a ajuda da Fig. 1 estas condições são descritas como se segue

a) Para evitar bloqueio é imposta a seguinte condição para o ponto no topo do subrefletor $\left(\mathrm{x}_{1}\right)$

$$
\mathrm{x}_{1}=\frac{\left[\tau_{1}\left(\mathrm{~s}_{1}-\ell_{\partial}\right)+\mathrm{x}_{\mathrm{E}} \ell\right]\left[\mathrm{s}_{1}\left(\ell_{0}+2 \mathrm{z}_{\mathrm{E}}-2 \mathrm{~s}_{1}\right) 1 \tau_{1}\left(\tau_{1}-\mathrm{x}_{\mathrm{E}}\right)\right]}{\left[\mathrm{s}_{1}^{2}\left(\ell_{0}+2 \mathrm{z}_{\mathrm{E}}-2 \mathrm{~s}_{1}\right)+\left(\tau_{1}-\mathrm{x}_{\mathrm{E}}\right)^{2} \ell_{0}-2 \mathrm{~s}_{1} \tau_{1}\left(\tau_{1}-\mathrm{x}_{\mathrm{E}}\right)\right]}
$$

a qual é obtida substituindo $M(\tau)$ e suas derivadas em $\tau=\tau_{1}$ na expressão (4) e denotando $\mathrm{s}_{1}=\mathrm{s}\left(\tau_{1}\right)$,

b) a fim de obter controle da iluminação de borda do subrefletor é imposta a seguinte condição para os raios incidentes no topo e base do subrefletor

$$
\eta_{c}=\tan \left(\theta_{c)}=\left|\left[\eta_{1} \eta_{2}-1\right] /\left[\eta_{1}-\eta_{2}\right]\right|\right.
$$

onde $\eta_{1}=\eta\left(\tau_{1}\right)$ e $\eta_{2}=\eta\left(\tau_{2}\right)$ definem as direções destes raios, e

c) o controle da dimensão $D_{s}$ do subrefletor no plano de simetria é imposta por

$$
\mathrm{x}_{2}=\mathrm{x}_{1}-\mathrm{D}_{\mathrm{s}}=\frac{\left[\tau_{2}\left(\mathrm{~s}_{2}-\ell_{\partial}\right)+\mathrm{x}_{\mathrm{E}} \ell_{o}\right]\left[\mathrm{s}_{2}\left(\ell_{0}+2 \mathrm{z}_{\mathrm{E}}-2 \mathrm{~s}_{2}\right)-\tau_{2}\left(\tau_{2}-\mathrm{x}_{\mathrm{E}}\right)\right]}{\left[\mathrm{s}_{2}^{2}\left(\ell_{0}+2 \mathrm{z}_{\mathrm{E}}-2 \mathrm{~s}_{2}\right)+\left(\tau_{2}-\mathrm{x}_{\mathrm{E}}\right)^{2} \ell_{0}-2 \mathrm{~s}_{2} \tau_{2}\left(\tau_{2}-\mathrm{x}_{\mathrm{E}}\right)\right]}
$$

sendo $s_{2}=s\left(\tau_{2}\right)$.

A solução do problema é obtida pela solução numérica iterativa deste sistema de três equações não-lineares em termos dos parâmetros $\mathrm{x}_{\mathrm{E}}, \mathrm{z}_{\mathrm{E}}$, e $\ell_{\sigma}$

\section{Soluções Aproximadas}

O sistema de equações não-lineares acima possue um conjunto de soluções matemáticas entre as quais podem existir soluções fisicamente não realizáveis. A adoção de técnicas numéricas iterativas implica que, tanto a estabilidade e a eficiência dos métodos, quanto a solução a ser obtida (dentre aquelas do conjunto) dependem de uma solução inicial adequada. A fim de visualizar as configurações de refletores possíveis, algumas aproximações podem ser introduzidas nas equações não lineares. 
Em geral, a exigência da utilização de uma geometria compacta força que $\tau_{1}$ e $x_{1}$ estejam próximos ao eixo-z. Isto permite assumir que $\left|\tau_{1}-x_{E}\right|<<R_{E}$, fazendo com que (7) e (3), para o raio que cruza a abertura em $\tau_{1}$, possam ser aproximadas por

$$
\begin{gathered}
\mathrm{x}_{1} \cong \tau_{1}-\ell_{o}\left(\tau_{1}-\mathrm{x}_{\mathrm{E}}\right) / \ell_{0} \quad \text { ou } \quad \mathrm{x}_{\mathrm{E}} \cong \tau_{1}-\mathrm{R}_{\mathrm{E}}\left(\tau_{1}-\mathrm{x}_{1}\right) / \ell_{0} \\
\mathrm{e} \quad \eta_{1} \cong\left[\ell_{0}+2\left(\mathrm{z}_{\mathrm{E}}-\mathrm{T}_{\mathrm{E}}\right)\right] / \mathrm{x}_{1}
\end{gathered}
$$

Substituindo (11) em (9), para o raio incidente no subrefletor em $\mathrm{x}_{2}$, obtem-se

$$
\mathrm{x}_{2} \cong \frac{\left[\tau_{2}\left(\mathrm{~s}_{2}-\ell\right)+\mathrm{x}_{\mathrm{E}} \ell\right]\left\{\mathrm{s}_{2}\left[\eta_{1} \mathrm{x}_{1}+2\left(\mathrm{R}_{\mathrm{E}}-\mathrm{s}_{2}\right)\right]-\tau_{2}\left(\tau_{2}-\mathrm{x}_{\mathrm{E}}\right)\right\}}{\mathrm{s}_{2}\left\{\mathrm{~s}_{2}\left[\mathrm{x}_{1} \eta_{1}+2\left(\mathrm{R}_{\mathrm{E}}-\mathrm{s}_{2}\right)\right]-\tau_{2}\left(\tau_{2}-\mathrm{x}_{\mathrm{E}}\right)\right\}-\left(\tau_{2}-\mathrm{x}_{\mathrm{E})}\left[\tau_{2}\left(\mathrm{~s}_{2}-l_{\partial}+\mathrm{x}_{\mathrm{e}} l_{a}\right]\right.\right.}
$$

A equação acima pode ser combinada com aquela para $\eta_{2}\left(\tau_{2}\right)$ dada por (3), resultando em

$$
\eta_{2}=\left[x_{1} / x_{2}\right] \eta_{1}+\left[\left[2 s_{2}\left(R_{E}-s_{2}\right)+\left(\tau_{2}-x_{2}\right)\left(\tau_{2}-x_{E}\right)\right] /\left(s_{2} x_{2)}\right]=a \eta_{1}+b\right.
$$

A análise de (13), para sistemas com folga $F \ll 2 R_{V}$, permite aproximar a diferença $\left(\tau_{2}-x_{E}\right)$ por $\left(\tau_{2}-x_{1}\right)$ em (13), fazendo com que os parâmetros a e b sejam independentes de $\mathrm{x}_{\mathrm{E}}, \ell_{0}, \mathrm{z}_{\mathrm{E}}$. Assim, a substituição de (13) em (8), resulta num polinômio do segundo grau em termos $\eta_{2}$ que tem as seguintes soluções

$$
\left.\left.\eta_{1}=\left\{-\left(b+n_{c}(1-a)\right) \pm\left(b+n_{c}(1-a)\right)^{2}-4 a\right)\right]^{1 / 2}\right\} /(2 a)
$$

A solução associada ao sinal (+) é desprezada pois requer um subrefletor largo e com o topo próximo à fonte pontual. Para a opção (-), o valor de $\eta_{1}$ pode ser aplicado em (10) e (12) permitindo, desta forma, obter $x_{E}$ e $l_{o}$. Os valores para $Z_{E}$ são obtidos substituindo $\ell_{0}$ em (11).

Quando $\mathrm{x}_{\mathrm{E}}=0$ (o caso axialmente simétrico), e assumindo $\tau_{2}<\mathrm{R}_{\mathrm{E}}$, $\ell_{0}$ é aproximado por $\ell_{0} \cong \mathrm{R}_{\mathrm{E}}\left(\mathrm{F} / \tau_{1}\right)$ e $(13)$ pode ser rescrita como

$$
\left.\eta_{2} \cong \eta_{1} s_{2} x_{1} /\left[t_{2}\left(s_{2}-\ell\right)\right]\right] \text { ou } \eta_{2} \cong a \eta_{1}
$$


a qual, quando substituída em (8), resulta em equação quadrática em termos de $\eta_{1}$, com soluções

$$
\left.\eta_{1}=\left\{-\eta_{C}(1-a) \pm(1-a) \eta_{C}-4 a\right]^{1 / 2}\right\} /(2 a)
$$

onde a opção de sinal esta associada aos comentários feitos anteriormente. Para os casos a serem analisados neste trabalho, as expressões aproximadas forneceram valores para $\left(\ell_{\sigma} \mathrm{x}_{\mathrm{E}}, \mathrm{z}_{\mathrm{E}}\right)$ que diferem de menos de $1 \%$ da solução do problema. Além de assegurar convergência para um número mais abrangente de casos e de aumentar a eficiência do processo numérico, estas aproximações permitem pré-selecionar uma das soluções.

\section{Análise dos Campos na Abertura de um Refletor Esférico}

Apesar da assimetria, sistemas de duplos refletores offset oferecem a possibilidade de ajustar a posição relativa das superfícies a fim de minimizar a componente de polarização cruzada na abertura do refletor principal ou eliminá-la completamente como no caso do sistema formado por cônicas confocais (Parabolóide-Hiperbolóide/Elipsóide). Para sistemas modelados para produzir fase uniforme, a polarização dos campos na abertura pode ser analisada com a ajuda da Referência [7], onde o autor relaciona a função $M(\tau)$ com a rotação $\psi_{0}$ do vetor polarização introduzida pela dupla reflexão. Isto é dado por

$$
\exp \left[-\mathrm{i} \psi_{0}\right]=\frac{\left.\left[\ell_{0} \mathrm{M}_{\tau}+\tau \mathrm{M}_{\bar{\tau}}+1\right)\right]}{\left[\ell_{0} \mathrm{M} \bar{\tau}+\eta_{0}\left(\bar{\tau} \mathrm{M}_{\tau}+1\right)\right]}
$$

onde $\eta_{0}=\cot \left(\theta_{0} / 2\right)$ está associada com o ângulo $\left(\theta_{0}\right)$ que o eixo do alimentador faz com o eixo-z (ver Fig.1).

Para sistemas com refletor principal esférico, esta expressão para $\psi_{0}$ pode ser obtida através da substituição da derivada de $\mathrm{M}$ ( $\tau$ ) (Eq.5) em (17), o que resulta em

$$
\exp \left[-\mathrm{i} \psi_{0}\right]=\frac{\left.\left.\{\bar{\tau} \mathrm{s}(\tau)-1)+\eta_{0} \mathrm{x}_{\mathrm{E}} \bar{\tau}+\eta_{0}|\tau|^{2}\{\mathrm{~s}(\tau)-1)+\mathrm{s}(\tau)\right]+\mathrm{x}_{\mathrm{E}}\right\}}{\left.\left.\{\tau \mathrm{s}(\tau)-1)+\eta_{0} \mathrm{x}_{\mathrm{E}} \tau+\eta_{0}|\tau|^{2}\{\mathrm{~s}(\tau)-1)+\mathrm{s}(\tau)\right]+\mathrm{x}_{\mathrm{E}}\right\}}
$$

Supondo um alimentador sem polarização cruzada segundo a terceira definição de Ludwig [8], a condição para que os campos sejam linearmente polarizados na abertura é obtida da imposição de que a parte imaginária da equação acima seja nula, resultando em 


$$
\operatorname{Im}\{\tau s(\tau)-1)+\eta_{0} x_{E} \bar{\tau}=0
$$

Esta condição imp1ica que a componente de polarização cruzada será nula sobre duas linhas no plano da abertura: o eixo x (sobre o plano de simetria da estrutura) e uma circunferência de raio

$$
\mathrm{R}_{\mathrm{XP}}=\left[\mathrm{R}_{\mathrm{E}}^{2}-\left(\ell_{0}-\eta_{0} \mathrm{x}_{\mathrm{E}}\right)^{2}\right]^{1 / 2}
$$

e centro sobre o ponto $\left(\mathrm{x}_{\mathrm{E}}, 0\right)$.

Para ilustrar a polarização dos campos na abertura, as Figuras 2.a e 2.b mostram respectivamente os valores de sen $\left(\psi_{0}\right)$ produzidos pelo projeto esboçado em [2] e por aquele obtido pelo ajuste dos parâmetros para que o círculo de polarização cruzada nula passe pela abertura. Como observado de (19) e pela comparação das figuras 2.a e 2.b, embora não seja possível eliminar completamente a rotação $\psi_{0}$, uma redução significativa nos níveis de polarização cruzada pode ser esperada quando o círculo passa pela abertura e, em especial sobre aquela área mais intensamente iluminada. Esta área está associada aos raios próximos ao eixo do alimentador na direção $\theta_{0}$. Assim, uma aproximação para a condição de mínima polarização cruzada pode ser escrita como

$$
\mathrm{x}_{0}\left(\eta_{0}\right)=\mathrm{x}_{\mathrm{E}}+\mathrm{R}_{\mathrm{XP}}
$$

onde $x_{0}\left(\eta_{0}\right)$ é o ponto na abertura mapeado pelo raio ao longo do eixo do alimentador. 


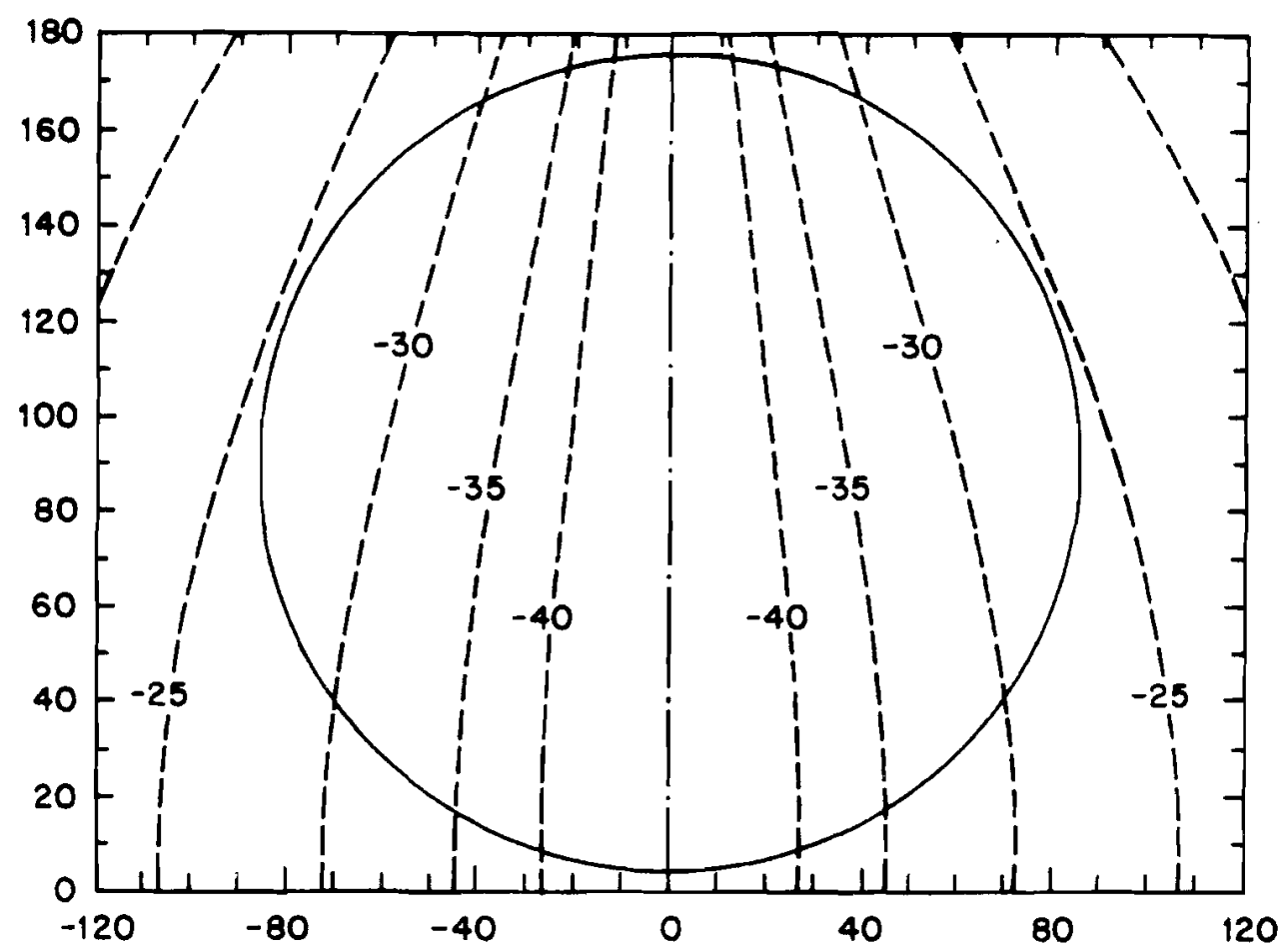

(a)

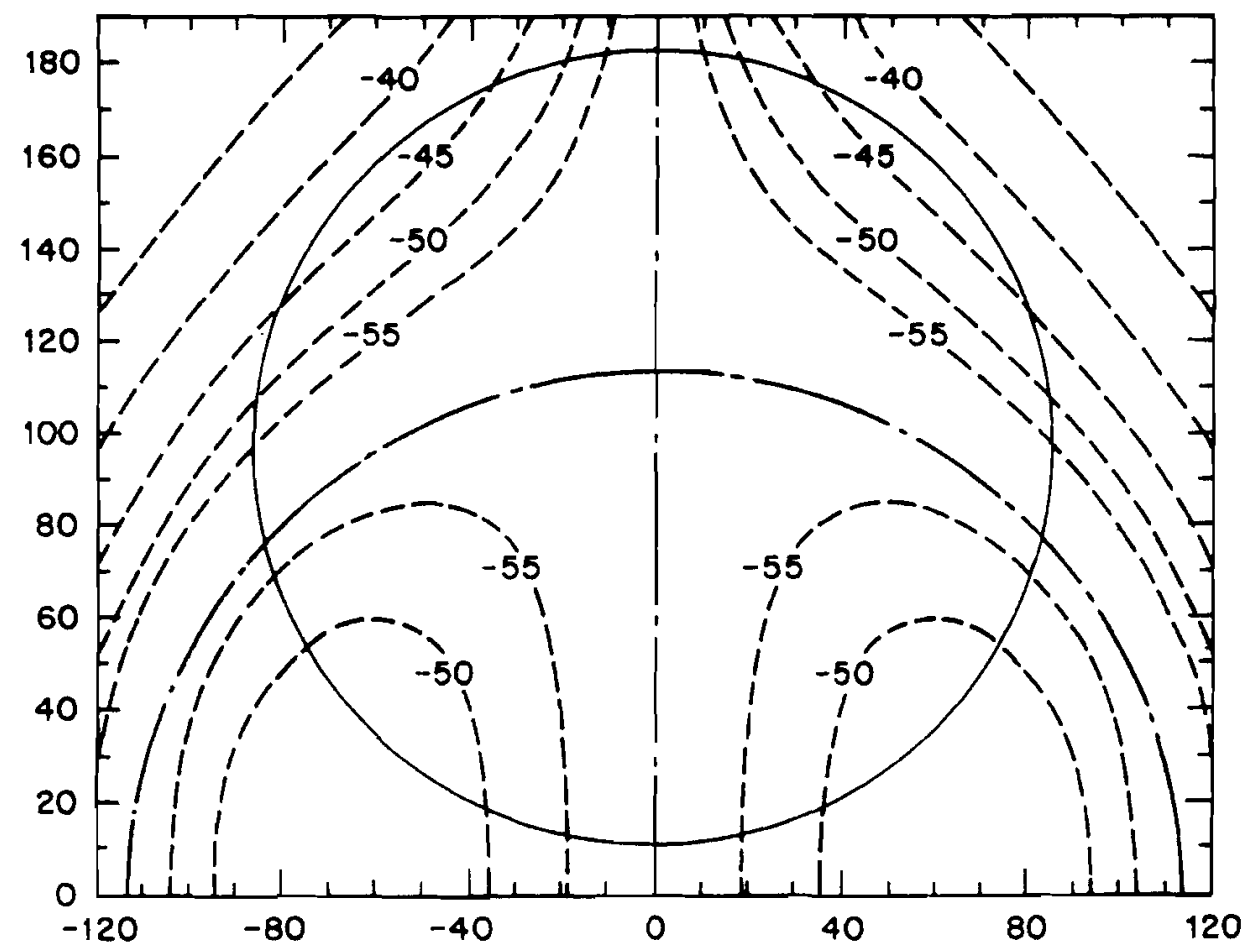

(b)

Figura 2 - Relação do vetor polarização $\psi_{0}$ representada por sen $\left(\psi_{0}\right)$ em dB(- - -). As linhas para $\psi_{0}=0^{0}$ são dadas por $\left(-^{\cdot} \cdot{ }^{-}\right)$e e o perímetro dado por ${ }($. 


\section{Projeto com Abertura Circular}

Como mencionado anteriormente, este trabalho tem como objetivo considerar modificações no projeto descrito em [2]. Naquele trabalho, o centro do esferóide esta colocado sobre o eixo- $z$ e a superfície do subrefletor é uma porção de uma superfície axialmente simétrica. Os refletores foram projetados impondo-se as duas primeiras condições (A) e (B) descritas pelas equações (7) e (8), não apresentando nenhum controle das dimensōes do subrefletor. Aqui, o centro do esferóide $\left(\mathrm{x}_{\mathrm{E}}, 0, \mathrm{z}_{\mathrm{E}}\right)$ pode ocupar qualquer posição ao longo do plano de simetria. Esta liberdade adicional é utilizada para impor, simultaneamente as condições (A) e (B) mencionadas anteriormente, uma terceira condição através da especificação da dimensão $\mathrm{D}_{\mathrm{s}}$ do subrefletor sobre o plano de simetria, a qual e expressa por (9).

Após o sistema de refletores ser definido pela solução do sistema de equações não-lineares em termos de $\left(\ell_{0}, z_{\mathrm{E}}, \mathrm{x}_{\mathrm{E}}\right)$, o ângulo do eixo do alimentador $\theta_{\mathrm{o}}$ é ajustado para otimizar o ganho da antena. Isto é obtido através de um compromisso entre a minimização das perdas por transbordamento e a minimização da distorção na distribuição de campo na abertura introduzida pela aberração esférica que produz uma concentração de potência no topo da abertura no caso de configuração gregoriana.

Para comparação, nossa investigação utiliza uma geometria com parâmetros idênticos aos utilizados na Referência [2]: abertura com diâmetro $\mathrm{D}=172 \lambda$, modelo de alimentador com diagrama ( $\cos ^{47} \theta$ ), e o semi-ângulo $\theta_{C}=25^{\circ}$ que foi escolhido para maximizar eficiência. Em contraste com o projeto apresentado em [2], uma folga $F=5 \lambda$ foi imposta ao projeto pois o topo do subrefletor não esta restrito a porção negativa do eixo-x. $O$ controle da folga $F$ e da dimensão $D_{s}$, impossível quando $x_{E}=0$, resulta em projetos verticalmente mais compactos. Para observar o efeito produzido por projetos com diferentes dimensões $D_{s}$ e raios do esferóide $R_{E}$, as Fig. 3.a-d mostram o desempenho para três casos representados pelos pares (Ds, RE). Os valores para o Caso I são aqueles utilizados em [2].

A análise é efetuada através do ajuste de $X_{E}$ para uma faixa de valores de $\tau_{c}$ que é limitada pelo bloqueio do alimentador e pela degradação do desempenho. A eficiência fornecida pela análise (Ótica Física) é mostrada em Fig. 3.a. Quando comparada com os valores encontrados em [2], a possibilidade de ajustar $\mathrm{X}_{\mathrm{E}}$ permite obter um aumento da eficiência para $69,5 \%$. Esta figura mostra também os valores de eficiência obtidos para geometrias empregando subrefletor com diversas dimensões $D_{s}$ e raio $R_{E}$. Para estas curvas, e observado que a diminuição das dimensões subrefletor $\mathrm{D}_{\mathrm{s}} \mathrm{e}$ a elevação do centro da abertura contribuem para aumentar a distorção da distribuição de campo na abertura e, conseqüentemente, para a redução da eficiência. É possível, também, observar que, embora resulte em geometrias menos compactas, a utilização de refletores com raio de curvatura $R_{E}$ maior pode compensar o acréscimo de distorção de potência associada a utilização de um subrefletor menor. A Figura 4 ilustra uma distribuição de potência típica na abertura de configurações gregorianas, correspondendo ao projeto mostrado na Fig. 2 para o Caso I $\operatorname{com} \tau_{\mathrm{c}}=90 \lambda$. 


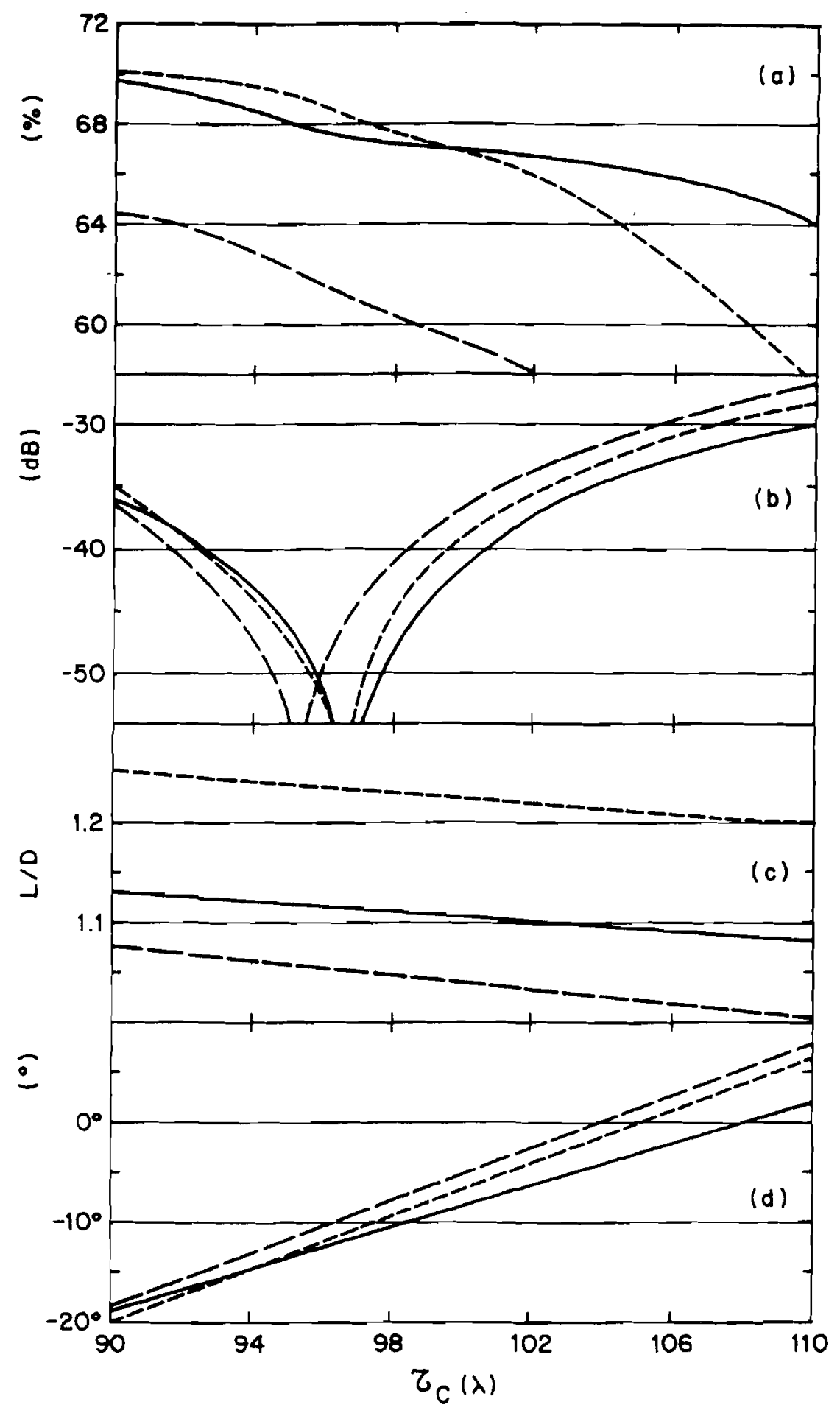

CASE I ( $D_{S}=0.25 D, R_{E}=344 \lambda$ )

CASE II $\left(D_{3}=0.2 D, R_{E}=344 \lambda\right)-$

CASE II $\left(D_{8}=0.2 D, R_{E}=344 \lambda\right)$

Figura 3 - Desempenho da configuração gregoriana com abertura circular 


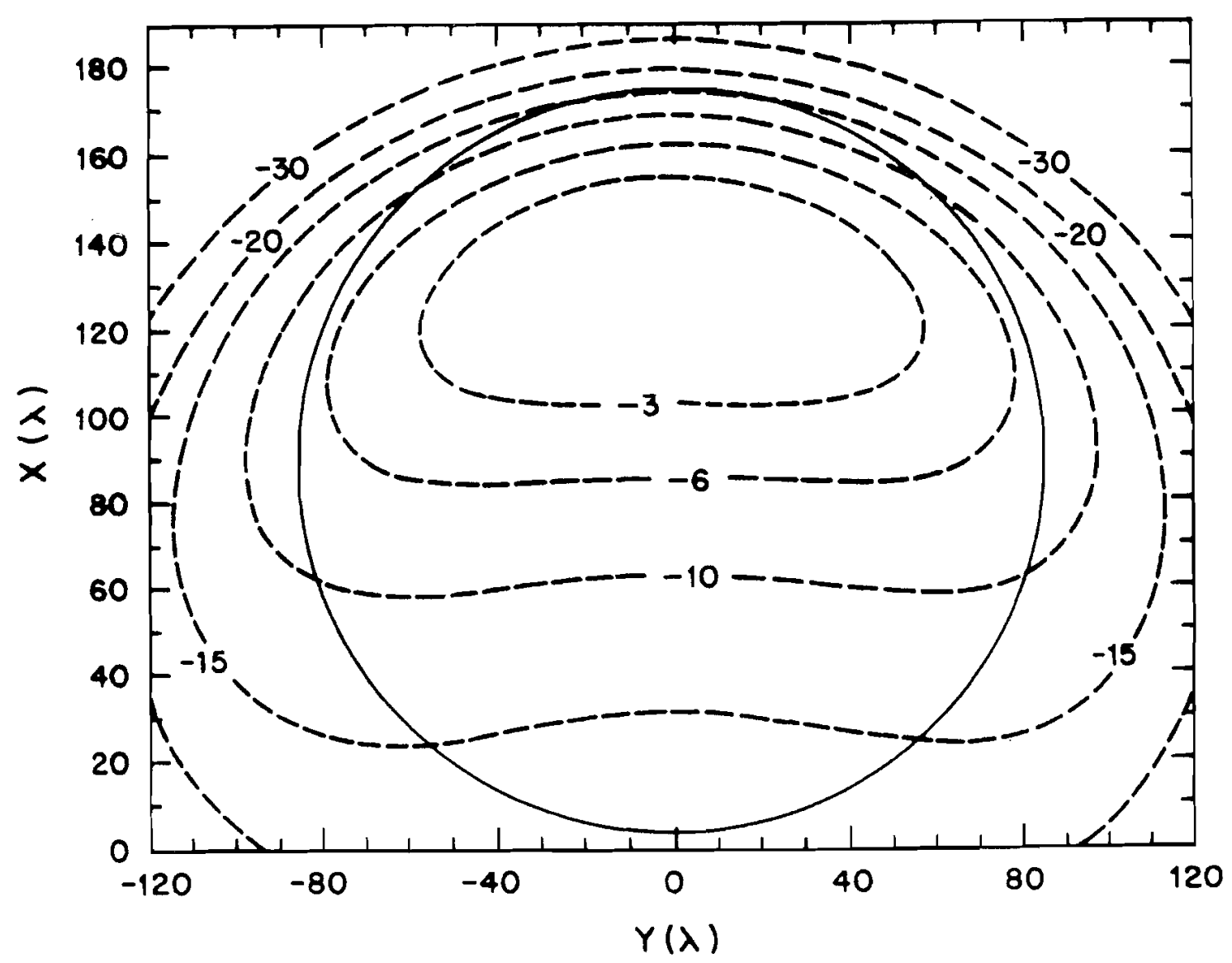

Figura 4 - Distribuição de potência na abertura circular produzido pelo projeto com $D_{S}=0.25 D, R_{E}=344 \lambda, Z_{C}=90 \lambda$ (Caso I). As linhas tracejadas (--.-) representam os contornos em dB referidos ao máximo e a linha $\leftrightharpoons$ representa o contorno da abertura.

Para a faixa de geometrias analisadas, a Fig. 3.b mostra os valores para pico de polarização cruzada em campo distante obtido da análise difrativa. Observa-se destes resultados que existe a possibilidade de ajustar a geometria para minimizar polarização cruzada, como foi demonstrado através da análise dos campos na abertura. O decréscimo no valor de pico mostrado na Fig. 4 corresponde aos projetos nos quais o círculo de polarização cruzada passa pela abertura. A busca por um valor apropriado de $\tau_{C}$ para o mínimo de polarização cruzada pode ser analiticamente efetuada adicionando-se uma quarta condição ao projeto, descrita por (21), como sugerido na seção anterior. Para os casos I, II, e III na Fig. 3.c, a utilização desta estratégia indica que os mínimos ocorrem para $\tau_{C}=96.7 \lambda, 95.2 \lambda$, e $96.0 \lambda$, respectivamente. Paralelamente aos valores de eficiência, a Fig. 3.d mostra o ângulo ótimo para $\left(\theta_{0}\right)$ obtido para cada uma das geometrias. Observa-se destas curvas que existe a possibilidade de ajustar o eixo do alimentador ao longo da direção do lóbulo principal. Embora isto implique a redução de ganho, esta possibilidade permite reduzir os efeitos do transbordamento no diagrama de radiação ao longo do plano de simetria. 


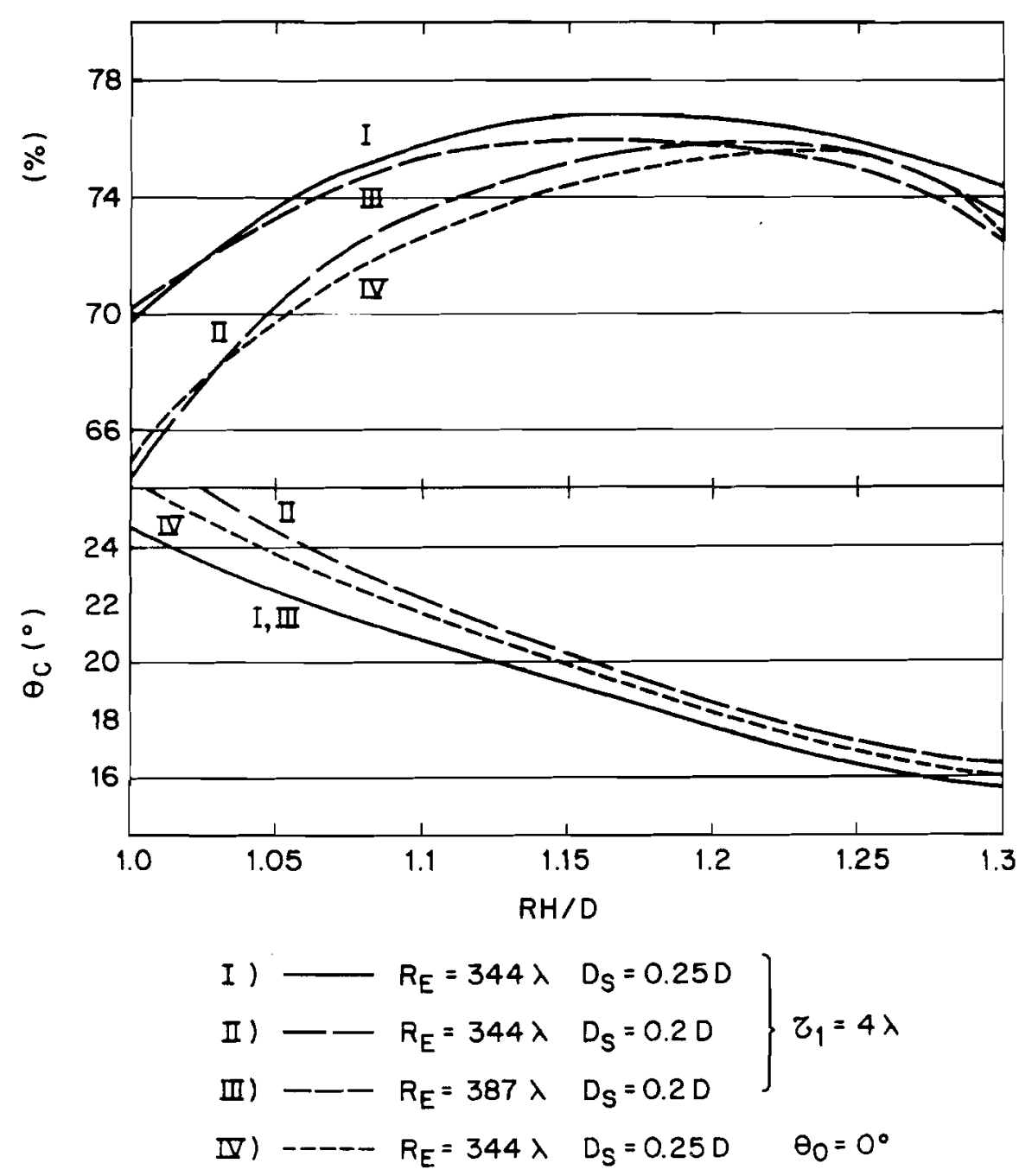

Figura 5 - Valores de eficiência (a) e semi-ângulo do cone de alimentação (b) para os projetos com abertura elíptica

\section{Projetos com Abertura Elíptica}

A fim de obter uma iluminação mais uniforme da abertura, a Fig. 4 sugere a utilização de uma abertura com perímetro elíptico. Para observar o comportamento da eficiência com o alongamento do perímetro, será considerado um conjunto de projetos com mesma área de abertura $\left(\pi R_{H} R_{V}=\pi D^{2} / 4\right)$ e iluminados pelo mesmo modelo de alimentador, onde os semi-eixos $R_{V}$ e $R_{H}$ são definidos na Fig. 1. Para cada valor de $\mathrm{R}_{\mathrm{H}} / \mathrm{D}$, o ângulo do cone $2 \theta_{\mathrm{C}}$ sobre o plano de simetria é ajustado para otimizar a eficiência. Para uma faixa de valores de $\mathrm{R}_{\mathrm{H}} / \mathrm{D}$, as Figs. 5.a e b ilustram os valores obtidos para a eficiência e para o ângulo ótimo $\theta_{\mathrm{C}}$. As curvas I, II, e III são geradas com os parâmetros dos projetos utilizados nas Figs. 3.a e b, mantendo-se a base da abertura constante em $\tau_{1}=4 \lambda$ de forma a evitar bloqueio por parte do subrefletor. 
Como observado na Fig.5.a, um considerável melhoramento no desempenho da antena pode ser obtido pelo alongamento da abertura. Para o caso I, a eficiência tem um máximo próximo de $76.8 \%$ quando $\mathrm{R}_{\mathrm{H}} / \mathrm{D} \cong 1.15$, representando um acréscimo de $13 \%$ quando comparado com os valores encontrados em [2]. Esta melhora é parcialmente devida ao melhor casamento entre a forma da abertura e os contornos da distribuição de campo. Paralelamente a isto, a uti1ização de dimensões $\mathrm{R}_{\mathrm{v}}$ menores permite abaixar o centro da abertura e, conseqüentemente, reduzir a distorção. Ao lado destes fatores, ao diminuir $\mathrm{R}_{\mathrm{v}}$, o ângulo de offset ótimo $\theta_{0}$ se aproxima do ângulo intermediário entre as direções $\eta_{1}$ e $\eta_{2}$, resultando em perdas menores por transbordamento e por difração na borda do subrefletor. Desta forma, como observado na Fig. 5.b, o alongamento da abertura permite estreitar $\theta_{C}$ e obter uma iluminação mais uniforme sobre a abertura. Todos estes fatores contribuem para diminuir a elipticidade das curvas de potência sobre a abertura como pode ser observado comparando-se as distribuições nas Figs. 4 e 6, correspondendo a última a geometria que produz os valores no topo da curva I na Fig. 5.

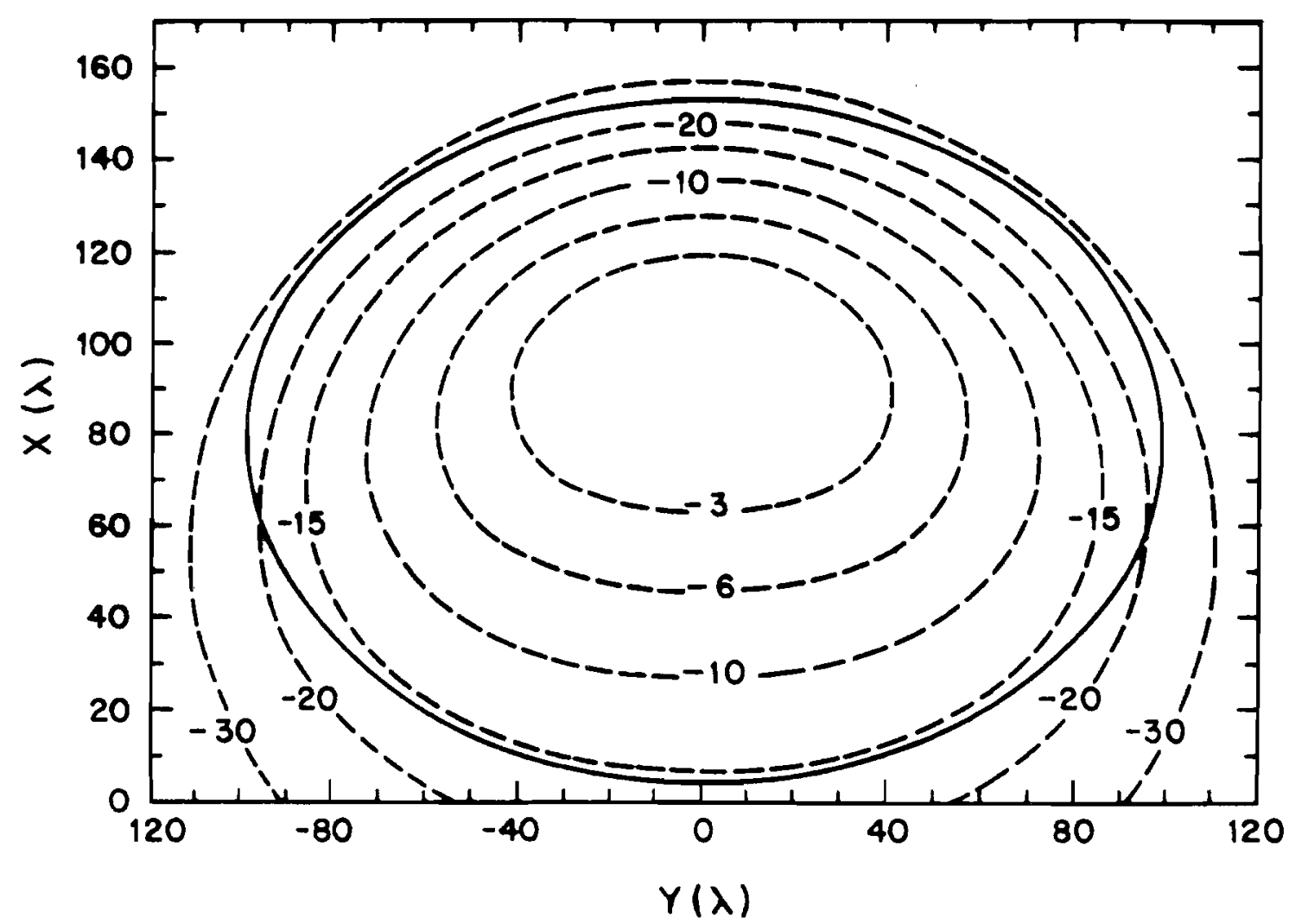

Figura 6 - Distribuição de potência na abertura para o projeto com abertura elíptica $\operatorname{com}\left(D_{S}=0.25 D, R_{E}=344 \lambda, Z_{C}=90 \lambda, R_{H} / D=1,15\right)$ (Casol). As linhas tracejadas (- - - ) representam os contornos em dB referidos ao máximo e a linha (representam o contorno da abertura. 
Como observado antes, o emprego de um subrefletor menor gera aumento da distorção, tornando a utilização da abertura elíptica mais efetiva, como pode ser verificado pelo considerável aumento na eficiência no caso II, quando comparado com os valores mostrados na Fig. 3.a. Para este caso, é encontrado um máximo de eficiência maior que $75 \%$ para $\left(\mathrm{R}_{\mathrm{H}} / \mathrm{D}\right) \cong 1.2$ (Casos I e III apresentam comportamento similar). O preço a ser pago pela utilização de aberturas elípticas no projeto é o aumento da dimensão Dy do subrefletor. Entretanto, este aumento em tamanho resulta em aumento da atenuação da iluminação de borda do subrefletor e, conseqüentemente, menor perda por transbordamento.

Sobre a abertura, isto implica, também, maior atenuação na iluminação do perímetro desta, em especial sobre o plano de assimetria, o que contribui para abaixar o envelope de lobos laterais da antena sobre este plano. Este efeito sobre o diagrama de radiação pode ser observado através da comparação das Figs. 4 e 6 onde o mínimo de atenuação sobre abertura vai das $-4 \mathrm{~dB}$ para $-9.5 \mathrm{~dB}$. Para o plano de assimetria, a Fig. 7 mostra uma comparação entre cortes dos diagrama de radiação resultantes da integração das correntes da Ótica Física obtidas para os projetos com abertura circular e elíptica.

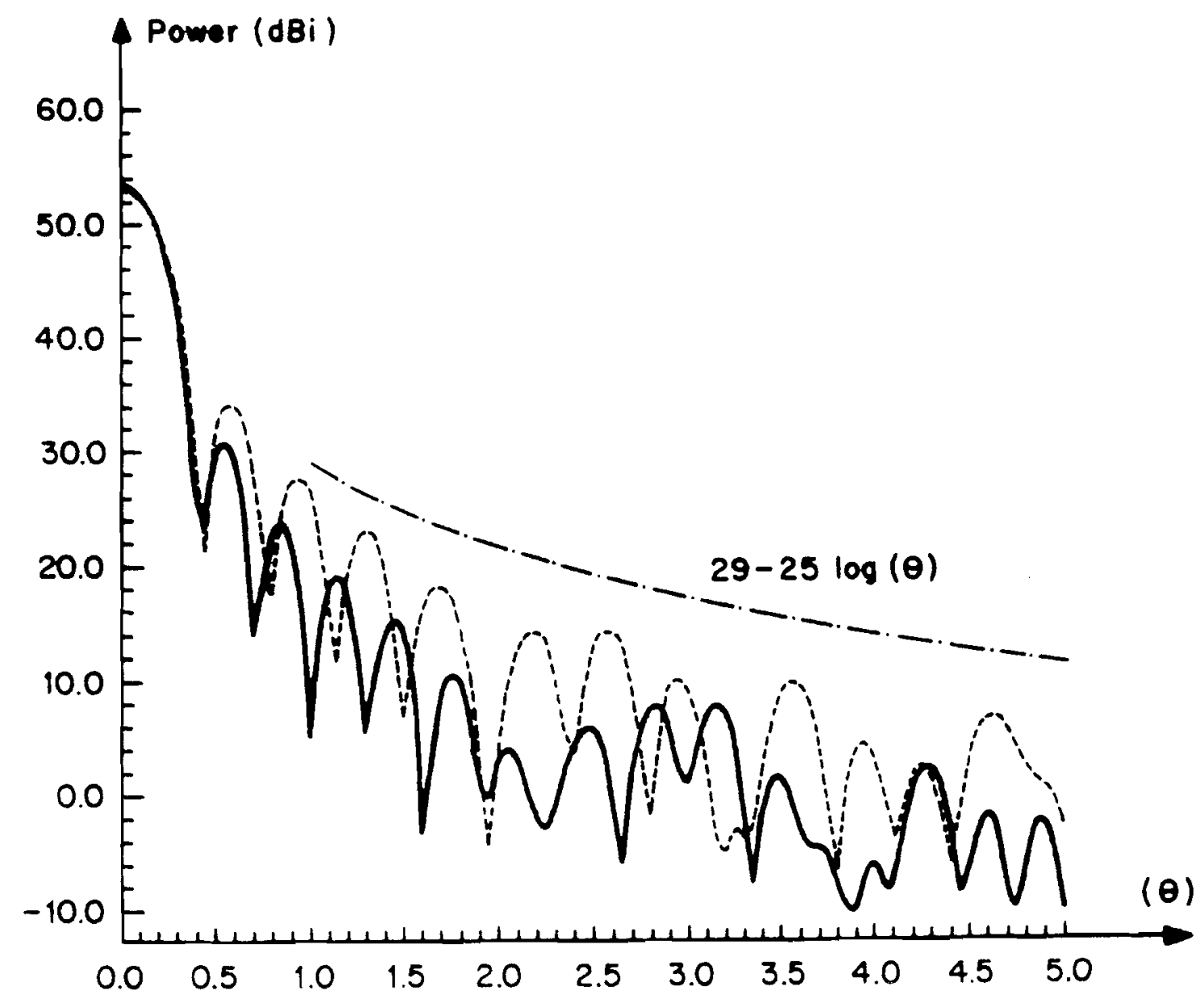

Figura 7 - Diagramas de radiação no plano de assimetria $\left(\phi=90^{\circ}\right)$ produzidos pelos projetos com abertura circular (- - -) e com abertura elíptica 
Observa-se uma redução de $6 \mathrm{~dB}$ para os lobos laterais próximos, assim como uma folga de $10 \mathrm{~dB}$ em relação as especificações. Como mostrado na Fig. 3, o ganho decresce com a elevação do centro da abertura. Para analisar o emprego de aberturas elíptica em projeto que requer a elevação do ponto do centro da abertura, será considerada a situação em que o eixo do alimentador e alinhado ao longo do lóbulo principal (eixo-z). A Curva IV na Fig. 4 foi gerada considerando-se diferentes valores para $\mathrm{R}_{\mathrm{H}} / \mathrm{D}$ e ajustando a geometria para se ter e $\theta_{0}=0^{\circ}$, assim como os valores de $\theta_{C}$, para maximizar a eficiência. Um pico de eficiência acima $75 \%$ foi encontrado para $\left(\mathrm{R} / \mathrm{D}_{\mathrm{H}}\right) \cong 1.25$, correspondendo a um acréscimo maior que aquele encontrado para a curva I. Isto mostra, também, que o alongamento da abertura é mais efetivo para projetos que requerem a elevação do centro da abertura. Outra situação interessante é descrita pela curva $\mathrm{V}$, a qual foi obtida através do ajuste simultâneo dos parâmetros $\left.\theta_{\mathrm{C}}, \mathrm{R}_{\mathrm{H}} / \mathrm{D}\right)$ para maximizar o ganho, e do centro da abertura, para minimizar polarização cruzada. Isto foi obtido incorporando na síntese a condição (quarta) dada por (21) ao lado de A, B, C, como descrito anteriormente. Para $\mathrm{D}_{\mathrm{S}}=0.25 \mathrm{D}$ e $\mathrm{R}_{\mathrm{E}}=344 \lambda$, a otimização dos parâmetros encontrou uma geometria com eficiência $76.1 \%$ para $\tau_{\mathrm{C}}=78,9 \lambda$ e com $\left(\mathrm{R}_{\mathrm{H}} / \mathrm{D}\right) \cong 1,2$. O máximo de polarização cruzada para este projeto está abaixo de $-60.0 \mathrm{~dB}$ como pode ser visto no corte do diagrama ao longo do plano de assimetria mostrado na Fig. 8. Os picos dos primeiros lobos laterais estão abaixo das especificações $(29-25 \log (\theta))$.

\section{Conclusões}

Neste trabalho, foi analisada a utilização de refletores esféricos em uma configuração de duplos refletores a serem empregados com antenas de pequenas estações terrestres de sistemas de comunicação via satélite. Duas novas características foram introduzidas no estudo: a utilização de aberturas elípticas e a relação da condição de simetria circular para o subrefletor. Como parâmetros de comparação de desempenho foi dada ênfase aos seguintes tópicos : máxima eficiência, baixa polarização cruzada, minimização do volume ocupado pela antena e menor subrefletor.

O desempenho de alguns projetos está sumarizado na Tabela 1, mostrando ser possível obter eficiência (Ótica Física) acima de $75 \%$ para sistemas com subrefletores menores que $0,25 \mathrm{D}$. Esta configuração permite alinhar o eixo do alimentador ao longo do lóbulo principal reduzindo perdas por transbordamento e seus efeitos no envelope de lobos secundários. Mostrou ser possível ajustar a posição relativa dos refletores, compensando a depolarização produzida pela dupla reflexão e minimizando a radiação de polarização cruzada. Foi mostrado que é possível obter projetos com polarização cruzada abaixo de -60, 0 dB sem degradação significativa da eficiência. 


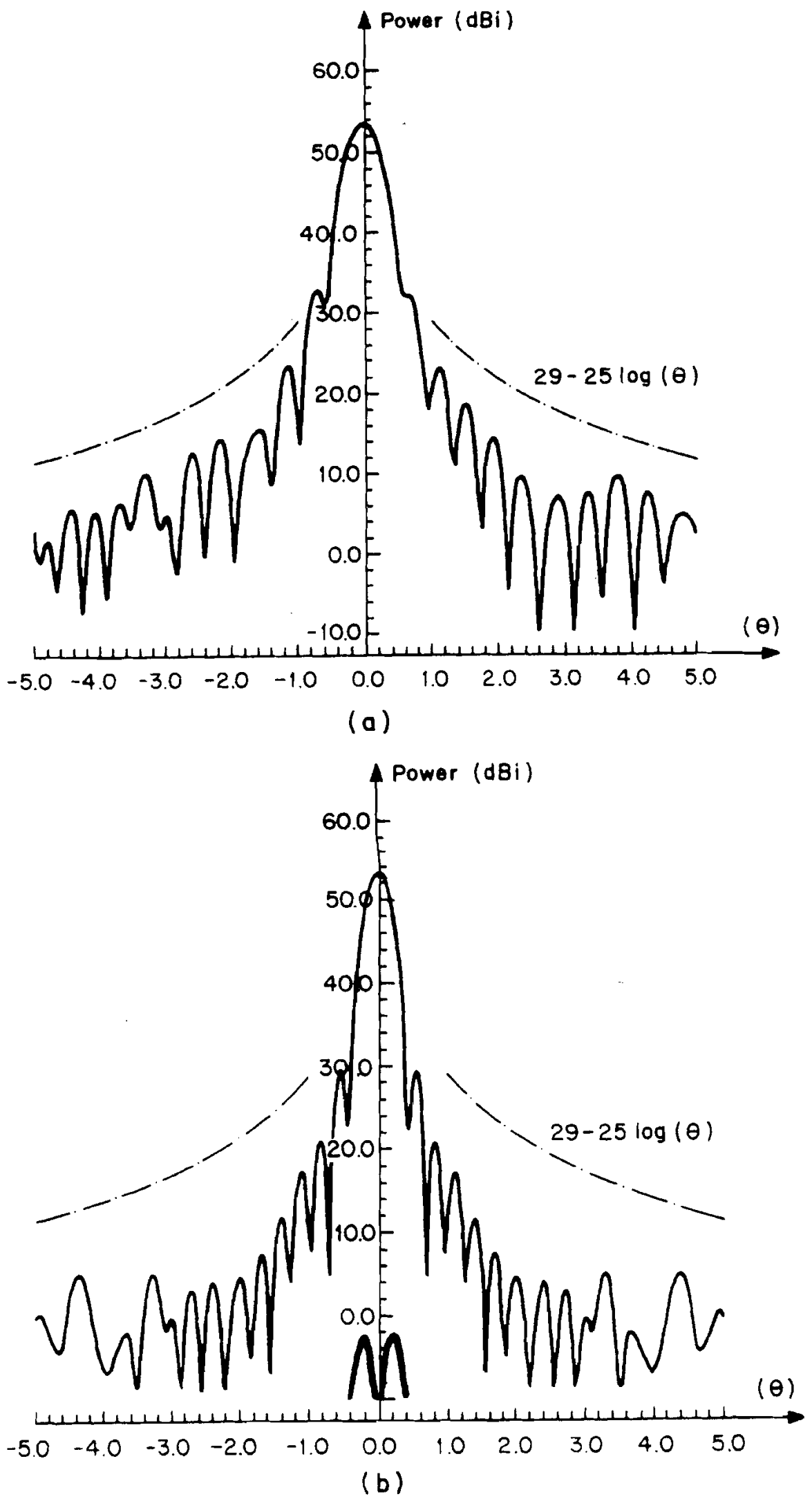

Figura 8 - Diagrama de radiação para projeto com baixa polarização cruzadas; (a) Plano de elevação (Plano-E); (b) Plano de assimetria (Plano-H) 
Tabela 1

Sumário dos resultados

\begin{tabular}{|c|c|c|c|c|c|c|}
\hline & $\operatorname{Ref}[2]$ & Caso I & Caso II & Caso III & Caso IV & Caso V \\
\hline & & . & $\tau_{1}=4 \lambda$ & \multicolumn{3}{|c|}{$\theta_{0}=0^{\circ}$} \\
\hline $\mathrm{R}_{\mathrm{H}} / \mathrm{d}$ & 1,0 & 1,15 & 1,2 & 1,15 & 1,25 & 1,2 \\
\hline$\theta_{\mathrm{C}}$ & 25 & 19 & 18 & 19 & 17 & 18 \\
\hline $\mathrm{D}_{\mathrm{y}} / \mathrm{D}$ & 0,219 & 0,302 & 0,267 & 0,243 & 0,356 & 0,325 \\
\hline $\mathrm{H} / \mathrm{D}$ & 1,30 & 1,15 & 1,06 & 1,10 & 1,08 & 1,11 \\
\hline $\mathrm{L} / \mathrm{D}$ & 1,09 & 1,194 & 1,15 & 1,302 & 1,195 & 1,197 \\
\hline Eficiência (\%) & 64,1 & 76,8 & 75,6 & 75,6 & 75,6 & 76,1 \\
\hline X-pol pico (dB) & $-38,4$ & $-36,2$ & $-35,2$ & $-36,2$ & $-32,3$ & $-64,1$ \\
\hline
\end{tabular}

\section{Referências}

[1] Cha, A.G.:“ An offset dual shaped reflector with 84.5. percent efficiency“, IEEE Trans., AP31, pp.896-902, 1983.

[2] Ramanujan, P., Clarricoats, P.J.B., E. Brown, R.C.: Offset spherical reflector with a low side lobe radiation", IEE Proc., Vol.134, Pt.H, pp.199-204, 1987

[3] Bjontegaard, G. E Petersen, T.:“ An offset dual reflector antenna shaped from near-field measurements of feed horn; theoretical calculations and measurements", IEEE Trans., AP-31, pp.973-977, 1983.

[4] Clarricoats, P.J.B., Brown, R.C., E Ramanujan, P.:" Comparative study of reflector antennas for small earth stations", IEE Proc., Part H, 134, pp.538-544, 1987

[5] Holt F.S. Bouchet, E.L.:" A gregorian corrector for spherical reflectors", IEEE Trans., AP12, pp.44-47, 1964.

[6] Chang, D. Rusch, W.V.T.:" An Offset-fed Reflector Antenna with an Axially Symmetric Main Ref1ector", IEEE Trans., AP-32, pp.1230-1236, 1984.

[7] Westcott, B.S.:' Shaped Ref1ector Antenna Design“, Research Studies Press, Letchworth, Herts., UK, 1983

[8] LUDWIG, A.C.:“ The definition of crosspo1arization”, IEEE Trans. AP, Vo1.AP-21, pp.116-119, 1973. 
José Ricardo Bergmann nasceu em Porto Alegre em 25 de março de 1953. Graduouse em Engenharia Elétrica pela Universidade Federal do Rio Grande do Sul em dezembro de 1975. Recebeu o título de Mestre em Engenharia Elétrica (MSc) pelo Instituto Militar de Engenharia em Fevereiro de 1979 e o título de Doutor em Engenharia Elétri$\mathrm{ca}(\mathrm{PhD})$ pela University of London em Dezembro de 1986. É professor associado da Universidade Católica do Rio de Janeiro, estando vinculado a esta universidade desde 1979. Seus principais interesses em pesquisa são eletromagnetismo aplicado, síntese e análise de antenas refletoras e antenas para comunicações via satélite. É atualmente presidente da Seção Rio de Janeiro do IEEE.

João Crisóstomo W. A. da Costa nasceu em Bragança, Piauí, em 17 de Janeiro de 1959. Graduou-se em Engenharia Elétrica pela Universidade Federal do Pará em dezembro de 1981. Recebeu o título de mestre em Engenharia Elétrica (MSc) pela Pontifícia Universidade Católica do Rio de Janeiro em Fevereiro de 1990 e Doutor em Engenharia Elétrica pela Universidade Estadual de Campinas em Abril de 1994. Esteve vinculado ao Departamento Nacional de Telecomunicações em Fortaleza entre 1982 a 1983 e Fundação de Telecomunicações do Pará entre 1984 e 1986. Atualmente, seus principais interesses são comunicações óticas, ondas milimétricas e estruturas seletivas de frequência. 